\title{
25.
}

\section{Enterprise Integration and Networking: Issues, Trends and Vision}

\author{
Arturo Molina ${ }^{1}$, David Chen ${ }^{2}$, Hervé Panetto ${ }^{3}$, Francois Vernadat ${ }^{4}$ and \\ Larry Whitman \\ IFAC TC 5.3 Enterprise Integration and Networking \\ 1CSIM-ITESM, armolina@itesm.mx \\ 2 UniversityBordeaux 1, chen@lap.u-bordeaux1.fr \\ 3 Université Henri Poincaré Nancy I, Herve.Panetto@cran.uhp-nancy.fr \\ 4 Eurostat, European Commission,.Francois.VERNADAT@cec.eu.int \\ 5 Wichita State University, larry.whitman@wichita.edu
}

\begin{abstract}
Enterprise Integration and Networking has been the topic of extensive research. Achievements deal with theoretical definition of reference models and architectures, modeling languages and tools, and development of relevant standards. The impact on today business has somehow been limited; therefore a revision of relevant issues and trends is required to establish a coherent vision for future research. This paper summarizes the underlying principles and challenges for enterprise modeling and integration, and its impact on enterprise networking.
\end{abstract}

\section{ENTERPRISE INTEGRATION AND NETWORKING CONTEXT}

The results of a study carried out in the United States for establishing the visionary manufacturing challenges for 2020 defined six grand challenges for manufacturers that represent gaps between current practices and the vision of manufacturing 2020 (NRC, 1998). These challenges are summarized in Table 1. In addition to these challenges, the Next Generation Manufacturing Systems (NGMS) will be more strongly time-oriented while still focusing on cost and quality. A NGMS should satisfy the following fundamental requirements:

- Enterprise integration and interoperability

- Distributed organization

- Model-based monitor and control

- Heterogeneous environments

- Open and dynamic structure

- Cooperation

- Integration of humans with software and hardware

- Agility, scalability and fault tolerance.

The requirements expressed above are related to the concept of the Networked Enterprise, be it an Extended, Fractal, Holonic or Virtual Enterprise (CamarinhaMatos et al., 1998) This is an emerging paradigm that results from the rapidly changing business environment forcing the complete supply chain from customers to suppliers to work in a more tightly-coupled mode. The Networked Enterprise relies 
to a large extent on Enterprise Integration and Enterprise Modeling techniques as defined in Vernadat (1996), Fox and Gruninger (1998) and Weston (1993).

Table 1. Manufacturing Challenges and Enterprise Integration proposals

\begin{tabular}{|c|c|c|c|c|c|c|}
\hline 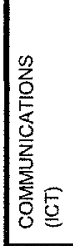 & 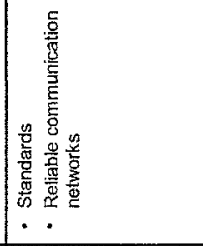 & 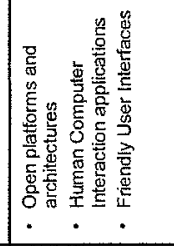 & 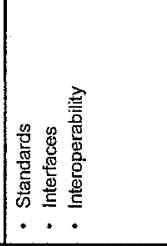 & 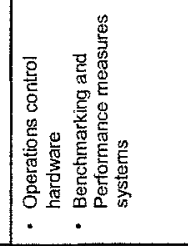 & 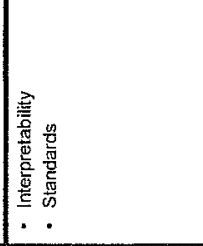 & 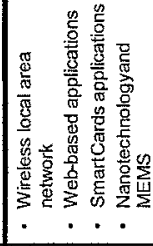 \\
\hline 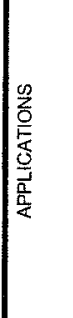 & 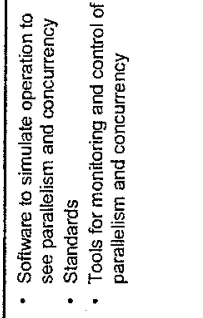 & 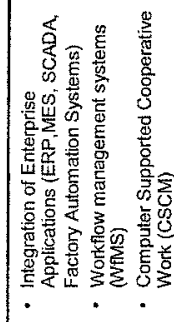 & 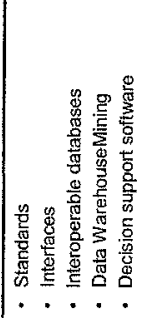 & 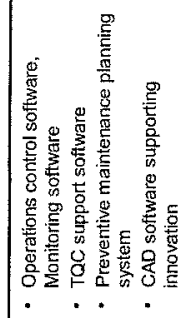 & 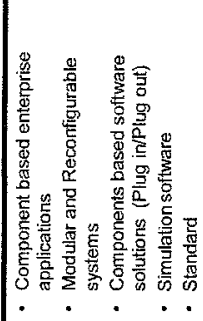 & 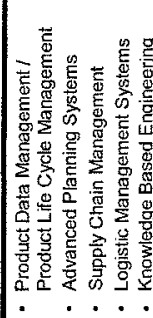 \\
\hline & 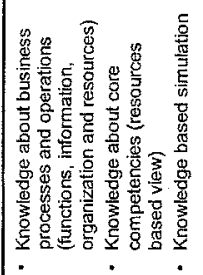 & 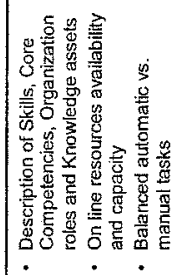 & 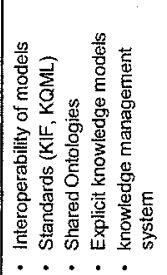 & 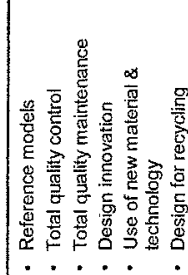 & 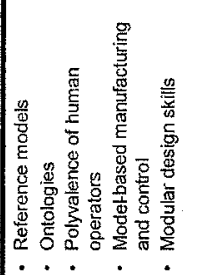 & 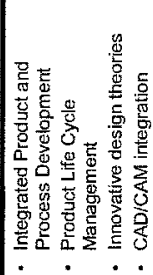 \\
\hline & 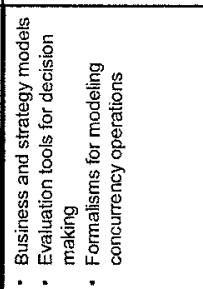 & 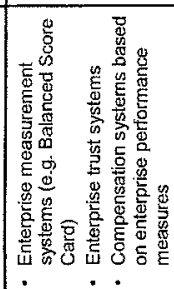 & 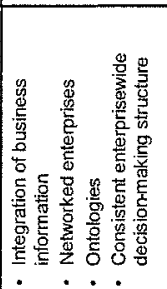 & 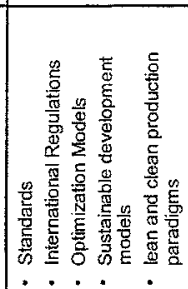 & 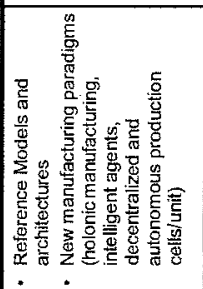 & 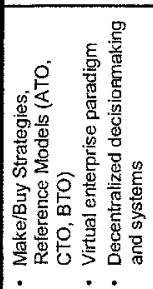 \\
\hline & 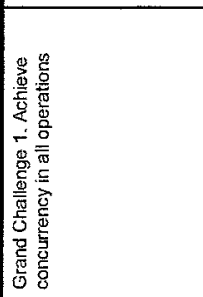 & 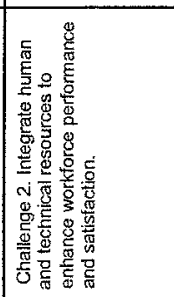 & 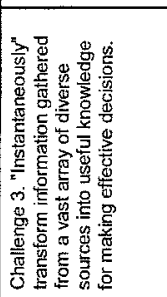 & 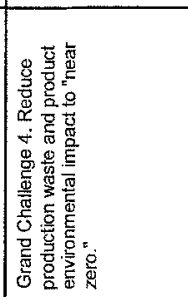 & 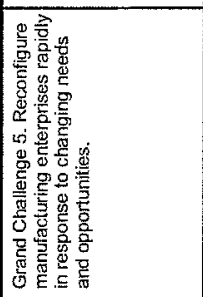 & 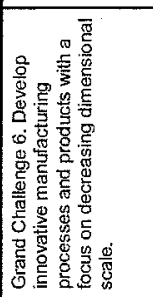 \\
\hline
\end{tabular}

Enterprise Integration and Modeling (EIM) enable an enterprise to share key data/information/knowledge in order to achieve business process coordination and cooperative decision-making, and therefore Enterprise Integration (Chen et al., 2001; Morel et al., 2003). Thus, there is a need for better process management and for more integration within individual enterprises and among networks of enterprises 
(Molina and Medina, 2003). The integration concept of providing quickly the right information at the right place at the right time under the right format throughout the enterprise is therefore evolving. Enterprise Integration now concerns (Vernadat, 2002; Whitman et al., 2001):

- Efficient business process management, integration and coordination;

- Enterprise-wide consistent decision-making;

- Team collaboration supported by Computer supported collaborative work (CSCW) for concurrent design and engineering activities;

- Increased flexibility throughout the company;

- Product life cycle management throughout the existence of a product;

- Interoperability of IT solutions, systems and people to face environment variability in a cost-effective way.

Recent advances in information and communication technologies have allowed manufacturing enterprises to move from highly data-driven environments to a more cooperative information/knowledge-driven environment. Enterprise knowledge sharing (know-how), common best practices use, and open source/web based applications are enabling to achieve the concept of integrated enterprise and hence the implementation of networked enterprises.

\section{NEEDS FOR ENTERPRISE INTEGRATION}

The question to answer is how Enterprise Integration and Modeling can deal with the technological challenges that allow an enterprise to face global competition and fluctuating market conditions. Using a reference model for Enterprise Integration, the contributions of the research area of Enterprise Integration and Networking can be classified into: Business, Knowledge, Application and Communications. Table 1 summarizes how the different challenges can tackle the issues faced by next generation manufacturing systems.

\subsection{Physical system integration, application integration, and business/knowledge integration}

The literature has reported that different forms of integration have emerged over the last decades. These being (Chen and Vernadat, 2004):

- Physical system integration (ICT),

- Application integration, and

- Business/Knowledge integration.

Physical system integration (Information and Communication Technologies) essentially concerns systems communication, i.e. interconnection and data exchange by means of computer networks and communications protocols. Physical system integration dates back to the early 1970's and is still evolving. Work done has first concerned the 7-layer OSU/ISO standard definition, and then the development of specialized manufacturing and office automation protocols such as MAP, TOP, and field-buses. It now continues with developments on ATM, fast Ethernet, Internet and web services, SOAP (Simple Object Access Protocol), or RosettaNet. Message queueing systems (such as IBM's MQ Series) and message-oriented middleware (MOM) are important corporate components of the basic infrastructure at this level. 
Application integration concerns interoperability of applications on heterogeneous platforms. This type of integration allows access to shared data by the various remote applications. Distributed processing environments, common services for the execution environment, application program interfaces (API's), and standard data exchange formats are necessary at this level to build cooperative systems. Application integration started in the mid 1980 's and is still on-going with very active work concerning STEP, EDI, HTML, XML, or eb-XML for the exchange of common shared data, development of common services for open systems around the web (web-services), integration platforms for interoperable applications in distributed environments (e.g. OSF/DCE, OMG/CORBA, WSDL, and more recently J2EE or Java to Enterprise Edition environments and .NET). Other tools used at this level are workflow management systems (WfMS) and computer support to collaborative work (CSCW).

Business/Knowledge integration relates to the integration at the corporate level, i.e. business process coordination, collaboration, knowledge sharing, and consistent enterprise-wide decision-making. This mostly concerns enterprise interoperability and requires externalizing enterprise knowledge to precisely model business operating rules and behavior. Early work has only been pursued by major programs financed by governments such as the ICAM and IPAD programs. More recently, the CALS Initiative and the Enterprise Integration Program (EIP) in the United States, as well as CIMOSA by the ESPRIT Consortium AMICE, GRAI decisional approach by LAP/GRAI of University of Bordeaux, AIT Initiative or the IST program of EU in Europe plus the Globeman Project of the IMS program investigated the issue.

\subsection{Business integration: towards the Networked Enterprise}

Enterprise Integration can be approached from five different perspectives, or levels, as shown in Table 2. At the sub-enterprise level, the functionality of the integrated application or system is limited to a relatively homogeneous area, typically a single local site under a single ownership. For example, flexible manufacturing systems are at the integrated sub-enterprise level. Complete functional integration at the singlesite enterprise level assures that business processes, manufacturing processes and product realization are united using a common architecture to fulfill a common goal. This is most likely for a single plant under single ownership, such as an automated factory.

The next three levels of EI - multi-site, extended, and virtual - occur over multiple geographic settings. Multi-Site enterprise integration is generally an issue faced by large enterprises (e.g., Boeing, IBM, General Motors, and EADS) in integrating heterogeneous systems throughout their facilities. An extended enterprise, which generally involves complex supply chains, concerns the integration of all members of the supplier and distribution chain to the common goal of market share capture through product realization. Virtual enterprises are very similar to extended enterprises, but they have the feature of being created and dissolved dynamically on a as-needed basis, and integration of member entities is largely electronic (Browne and Zhang 1999). All levels, to varying degrees, influence and are influenced by integrated product realization, integrated business systems, and tools enabling integration. While the objective is to support creation and operation of extremely efficient, flexible, and responsive extended 
manufacturing enterprises, the path to reach this will require capturing the wisdom achieved at each of the enterprise integration levels (Panetto et al., 2004).

Table 2. Levels of Enterprise Integration

\begin{tabular}{|l|l|l|l|l|l|}
\hline $\begin{array}{l}\text { Level of } \\
\text { Integration }\end{array}$ & Functionality & Geographic & Ownership & $\begin{array}{l}\text { Homogeneity of } \\
\text { Functional } \\
\text { Systems }\end{array}$ & \multicolumn{1}{|c|}{$\begin{array}{l}\text { Stage of } \\
\text { Maturity }\end{array}$} \\
\hline $\begin{array}{l}\text { Sub- } \\
\text { Enterprise }\end{array}$ & Limited & Local & $\begin{array}{l}\text { Single } \\
\text { Owner }\end{array}$ & Homogeneous & $\begin{array}{l}\text { State of } \\
\text { Industry }\end{array}$ \\
\hline $\begin{array}{l}\text { Single-Site } \\
\text { Enterprise }\end{array}$ & Complete & Local & $\begin{array}{l}\text { Single } \\
\text { Owner }\end{array}$ & Homogeneous & Leading Edge \\
\hline $\begin{array}{l}\text { Multi-Site } \\
\text { Enterprise }\end{array}$ & Complete & Distributed & $\begin{array}{l}\text { Single or } \\
\text { Multi- } \\
\text { Owner }\end{array}$ & Mixed & Leading Edge \\
\hline $\begin{array}{l}\text { Extended } \\
\text { Enterprise }\end{array}$ & $\begin{array}{l}\text { Static } \\
\text { complete }\end{array}$ & Distributed & $\begin{array}{l}\text { Multi- } \\
\text { Owner }\end{array}$ & $\begin{array}{l}\text { No, but may be } \\
\text { mixed in some } \\
\text { functions }\end{array}$ & Leading Edge \\
\hline $\begin{array}{l}\text { Virtual } \\
\text { Extended } \\
\text { Enterprise }\end{array}$ & $\begin{array}{l}\text { Dynamic } \\
\text { Complete }\end{array}$ & Global & $\begin{array}{l}\text { Multi- } \\
\text { Owner }\end{array}$ & No & $\begin{array}{l}\text { Limited in } \\
2004 \\
\text { More pervasive } \\
\text { in } \\
2015\end{array}$ \\
\hline
\end{tabular}

\section{ENTERPRISE MODELING AS THE MEANS TO ACHIEVE INTEGRATION}

Collaboration and coordination between people, applications, and computer systems require models that are shared among all the actors in a cooperative environment. Therefore, enterprise models are a must in achieving enterprise integration (Chen et al., 2002a; Whitman and Huff, 2001, Vernadat, 1996).

A core concept in enterprise modeling is the business process that encapsulates all the key elements of the enterprise, i.e. activities or functions, data/information/knowledge, human and technological resources. When a business model is created a representation of people interactions, roles and responsibilities, data/information exchange, resources required to execute certain activities, and procedures/instructions used to control functions are described in detail. The enterprise model is used as a semantic unification mechanism, or knowledge mapping mechanism, built by applying principles and tools of a given enterprise modeling method. Semantic concept definitions in the model can be expressed in the form of ontology, i.e. using a shared neutral knowledge representation format. The obtained enterprise model is also a means to represent shared concepts at a high level of abstraction and to capture stakeholders requirements (Panetto, 2001; Vernadat, 2002; Panetto et al., 2003).

The aim of Enterprise Modeling is to provide:

- A visualization of enterprise knowledge to better understand how an enterprise is structured and how operates; 
- Support change management using an enterprise engineering approach supported by structured analysis, rigorous design methods, simulation tools, and systematic decision-making; and

- A model used to control and monitor enterprise operations.

The main motivations for Enterprise Modeling are:

- Understanding how an enterprise is structured and behaves in order to manage system complexity,

- Capitalization of enterprise knowledge (know-what, know-how and know-why),

- Business process management based on enterprise engineering concepts,

- Improved change management in all types of processes,

- Achievement of enterprise integration and interoperability

The aims of Enterprise Integration are:

- To assist in the fulfillment of enterprise goals through the alignment of strategies, competencies and technologies in a company,

- To support the execution of collaborative, concurrently and distributed business processes,

- To enable business communication and coordination among various organizational entities of the extended enterprise,

- To facilitate knowledge sharing and information exchange between people and applications,

- To provide interoperability among heterogeneous, remotely located, independent vendor applications and Information and Communication Technologies (ICT).

\section{TRENDS AND FUTURE VISION}

\subsection{Concerning Enterprise Modeling and Reference Models}

New advances in Enterprise Engineering methods as well as a strong need to progress towards Enterprise Integration call for efficient enterprise modeling languages and advanced computer-based tools. Enterprise modeling is concerned with representation and analysis methods for design engineering and automation of enterprise operations at various levels of detail (e.g. coarse modeling, reengineering, detailed design and analysis, performance evaluation, etc.).

The following methods or architectures for enterprise modeling are considered key in this evolution: IDEFX (Whitman et al., 1997), GRAI-GIM (GRAI Integrated Methodology) (Doumeingts and Vallespir, 1995), CIMOSA (CIM Open System Architecture) (Berio and Vernadat, 1999), PERA (Purdue Enterprise Reference Architecture) (Williams, 1994), GERAM (Generalized Enterprise Reference Architecture and Methodology) (IFAC-IFIP Task Force, 1999; Bernus and Nemes, 1996).

Various methods and modeling techniques have been proposed over the last decade to cover different aspects of enterprise modeling, e.g., ARIS ToolSet, BONAPART, CimTool, FirstSTEP, IDEF methods, IEM, IBM's FlowMark, IMAGIM, METIS, PrimeObject and PROPLAN, to name a few.

The interoperability between these methodologies and tools is low. Most of the enterprises modeling tools are just graphical model editors. There are no model analysis functions and rules built in these tools. The proliferation of fancy and non inter-operable EM tools on the marketplace has created a prejudicial Tower of Babel 
situation. Unified languages are therefore proposed as consensus such as PSL (Process Specification Language) for manufacturing processes, supported by NIST in the US, or UEML (Unified Enterprise Modeling Language) for business processes, supported by EU (Panetto, 2002; Panetto, 2004; Vernadat, 2002). Also, while EM is widely used for well-structured processes, few EM tools deal with semi-structured or non-structured processes. Further developments are necessary in this area to better take into account human and organizational aspects. It is important to mention that Enterprise Modeling largely remains a concept or is even completely ignored by most SMEs (Small and Medium Enterprises). Various efforts are underway by standardization groups to propose standards (CEN/ISO WD 19440, ISO DIS 14258, ISO CD 15704, ODP Enterprise language and OMG ManTIS Task Force). Future trend in this area is to continue to develop UEML and to accelerate the standardization effort. For instance, the ISO TC184 SC5/WG1 is considering launching a new work item on process modeling language.

Concerning the reference architectures, literature survey shows that there are two types of architectures. Type 1 describes an architecture or physical structure of some component or part of the integrated system such as the computer system or the communications system. Type 2 presents an architecture or structure of the project which develops the physical integration, i.e., those that illustrate the life cycle of the project developing the integrated enterprise. Today, the architecture concept is not sufficiently exploited. One of the reasons is the lack of proper architecture representation formalism supporting significant characterization of features and properties of enterprise systems. Furthermore, existing architecture principles were not developed to a satisfactory level to allow bringing significant improvement to enterprise architecting. Further research is needed in this area.

Regarding reference models and in the area of standardization, some partial approaches can be mentioned. For examples, ISO 15531 MANDATE is a reference model focusing on information and resource views of manufacturing domain; the IEC 62264 series standard is a reference model on production management and control focusing on the information flow between the control domain and the rest of the enterprise. All these approaches are still on-going works and not mature. More recently, a European Technical Specification (CEN TS 14818: Decisional Reference Model) has been approved. It is based on the GRAI approach and shows a basic decision-making structure defined at high level abstraction.

\subsection{Concerning Enterprise and Processes Models Interoperability}

A significant initiative to develop interoperability between process models is ISO CD 18629 - Process Specification Language (PSL). In PSL a formal semantic approach (called PSL ontology) is used. However, important efforts are still needed to get effective implementation in industry. Another relevant initiative is the standard dealing with manufacturing software capability profiling (ISO 16100) carried out by ISO TC184/SC5/WG4.

The standard IEC/ISO 62264 (2002) defines models and establishes terminology (semantics) for defining the interfaces between an enterprise's business systems and its manufacturing control systems. It describes in a rather detailed way the relevant functions in the enterprise and the control domain and the objects normally exchanged between these domains. It is becoming the accepted model for B2M integration and interoperability. 
To meet new industrial challenges, there is a shift from the paradigm of total integration to that of interoperation. Relevant standardization activity focusing on interoperability is just starting and most of work remains to be done in the future.

In order to reach a broad consensus for model information exchange between enterprise modeling tools, the UEML project (Panetto et al., 2003a; Vernadat, 2002) has defined an initial set of generic constructs with the aim of achieving interoperability between them. In recent years, one of the most notable research efforts has been directed to improvement of interoperability (mainly software interoperability), a critical success factor for enterprises striving to become more flexible and to reduce the effort required to establish and sustain cooperation. Software interoperability has been especially addressed by specific software markets such as EAI and XML based solutions. However, these solutions mostly focus on compatibility of distinct formats without looking at the so-called modeling domain, i.e., the domain stating the rationale behind the software and providing reasons for building software. Information about the modeling domain, without taking into account any software issues, is essential to achieving greater interoperability. It is likely to be really difficult or even impossible to understand and recover this kind of information from software. As a consequence, this information should be associated with the software from the beginning and should be continuously maintained.

UEML could solve the issue of horizontal interoperability at the enterprise level. Thus, as information is controlled at the Automation level, it should need to be defined through a vertical interoperability approach from the product that produces it through the Manufacturing Execution System that consolidates it to the Enterprise Business Processes that use it. Standards such as the IEC/ISO 62264 together with the IEC 61499 function block draft standard for distributed industrial-process measurement and control systems could partially solve the vertical interoperability problem from the Business to the Manufacturing levels.

Consequently, as a prerequisite to building such a vertical information system dealing with physical process constraints, the TC5.3 UEML working group is aiming at defining and formalizing a practical and pragmatic language that should serve as a pivotal language ensuring a common understanding of the product information along its whole life cycle (Panetto et al., 2003b). At the European level, the INTEROP network of excellence will further develop UEML v1.0 and deliver a extended UEML specification v2.0. ATHENA Integrated Project and in particular project A1 will use UEML 1.0 as a baseline to develop a set of modeling constructs for collaborative enterprise. Applying AUTO-ID (Morel et al., 2003), that information can be embedded in physical objects according to the HMS (Holonic Manufacturing System) paradigm, in order to ensure the traceability of customized products, goods for manufacturing issues and services for logistics issues. Such a holonic approach requires aggregating separate object views and constructs of the IEC/ISO 62264 standard in order to define the relevant holons field, lack of established standards, which sometimes happen after the fact, and the rapid and unstable growth of the basic technology with a lack of commonly supported global strategy.

\subsection{Concerning Enterprise Integration of Applications and ICT}

Enterprise Integration (EI) is also becoming a reality for many companies, especially networked companies or enterprises involved in large supply-chains (extended or 
virtual enterprises). Some major projects for enterprise integration have been conducted in Europe (AIT Initiative) or in the US (EIF, NIIIP, NGM). The problem is that EI is both an organizational problem as well as a technological problem. The organizational problem is still partially understood so far. The technological problem has been the focus of major advances over the last decade, mostly concerning computer communications technology, data exchange formats, distributed databases, object technology, Internet, object request brokers (ORB such as OMG/CORBA), distributed computing environments (such as OSF/DCE and MS DCOM), and now J2EE (Java to Enterprise Edition and Execution Environments), .NET, and Web services. Some important projects having developed integrating infrastructure (IIS) technology for manufacturing environments include (Weston, 1993; Goranson et al., 2002; Morel et al., 2003):

- CIMOSA IIS: Integrating Infrastructure of CIMOSA

- AIT IP: This is an integration platform developed as an AIT project and based on the CIMOSA IIS concepts

- OPAL: This is also an AIT project that has proved that EI can be achieved in design and manufacturing environments using existing ICT solutions

- NIIIP (National Industrial Information Infrastructure Protocols)

More recently, research programs such as the ATHENA Integrated Project and the INTEROP Network of Excellence funded by E.C. in Europe look for innovative solutions regarding interoperability between legacy systems. However, EI suffers from inherent complexity of the field, lack of established standards, which sometimes happen after the fact, and the rapid and unstable growth of the basic technology with a lack of commonly supported global strategy. Nevertheless, EI must be seen as a goal, not a solution, to continuously progress towards a more integrated enterprise.

\section{CONCLUSIONS}

It is the author's opinion that Enterprise Modeling and Integration is slowly but surely becoming a reality. However, these technologies would better penetrate and serve any kind of enterprises if:

- There was a standard vision on what enterprise modeling really is and there was an international consensus on the underlying concepts for the benefit of business users (Goranson et al., 2002)

- There was a standard, user-oriented, interface in the form of a unified enterprise modeling language (UEML) based on the previous consensus to be available on all commercial modeling tools (Chen et al., 2002b; Panetto et al., 2004)

- There were real enterprise modeling and simulation tools commercially available taking into account function, information, resource, organization, and financial aspects of an enterprise including human aspects, exception handling, and process coordination. Simulation tools need to be configurable, distributed, agent-based simulation tools (Vernadat and Zeigler, 2000)

- There were design patterns and model-based components available as (commercial) building blocks to design, build, and reengineer large scale systems (Molina and Medina, 2003) 
- There were commercially available integration platforms and integrating infrastructures (in the form of packages of computer services) for plug-and-play solutions (Chen and Doumeingts, 2003)

Future trends in enterprise integration and enterprise modeling would be toward loosely-coupled interoperable systems rather than high-cost monolithic solutions and low-success holistic integration projects.

\section{REFERENCES}

Berio, G., Vernadat, F. (1999) New Developments in Enterprise Modeling Using CIMOSA. Computers in Industry, 40(2-3), 99-114.

Bernus, P., Nemes, L. (1996) Framework to define a generic enterprise reference architecture and methodology. Comp. Integrated Manuf. Systems. 9(3) 179-191.

Browne J., and Zhang J., (1999). Extended and virtual enterprises - similarities and differences, International Journal of Agile Management Systems, 1/1, pp. 30-36.

Camarinha-Matos LM, Afsarmanesh H, Garita C. (1998). Towards an architecture for virtual enterprises, J of Intelligent Manufacturing, 9 (2), 189-199.

Chen, D. and Doumeingts, D. (2003) European Initiatives to develop interoperability of enterprise applications - basic concepts, framework and roadmap. Journal of Annual reviews in Control, 27(3), 151-160.

Chen, D., and Vernadat, F. (2004). Standards on enterprise integration and engineering - A state of the art. International Journal of Computer Integrated Manufacturing, 17(3), 235-253.

Doumeingts, G. Vallespir, B. (1995). A methodology supporting design and implementation of CIM systems including economic evaluation. Optimization Models and Concepts in Production Management (P. Brandimarte and A. Villa, eds.), Gordon \& Breach Science Publishers, New York. pp. 307-331.

Fox, M.S., Gruninger, M. (1998) Enterprise Modeling. AI Magazine, Fall. 109-121.

Goranson, T., Jochem, R., Nell, J., Panetto, H., Partridge, C., Sempere Ripoll, F., Shorter, D., Webb, P., Zelm, M. (2002). New Support Technologies for Enterprise Integration. IFIP International Conference on Enterprise Integration and Modeling Technology (ICEIMT'02), Kluwer Academics Publisher, Valencia, Spain, 24-26 April 2002, pp. 347-358.

IFAC-IFIP Task Force (1999). GERAM: Generalized Enterprise Reference Architecture and Methodology, Version 1.6.3, IFAC-IFIP Task Force on Architecture for Enterprise Integration.

Molina, A., Sanchez, J.M., Kusiak, A. (1999). Handbook of Life Cycle Engineering: Concepts, Models and Technologies, Kluwer Academic Press.

Molina, A., and Flores, M., A. (1999). Virtual Enterprise in Mexico: From Concepts to Practice. Journal of Intelligent and Robotics Systems. 26: 289-302.

Molina, A. and Medina, V. (2003). Application of Enterprise Models and Simulation Tools for the evaluation of the impact of best manufacturing practices implementation. IFAC Annual Reviews in Control. 27 (2), 221-228.

Morel, G., Panetto, H., Zaremba, M-.B., Mayer, F. (2003). Manufacturing Enterprise Control and Management System Engineering: paradigms and open issues. IFAC Annual Reviews in Control. 27 (2), 199-209.

National Research Council. (1998). Visionary Manufacturing Challenges for 2020, Visionary manufacturing challenges for 2020 / Committee on Visionary 
Manufacturing Challenges, Board on Manufacturing and Engineering Design, Commission on Engineering and Technical Systems, National Academy Press. (http://search.nap.edu/readingroom/books/visionary/index.html)

Panetto, H. (2001). UML semantics representation of enterprise modeling constructs. Proceedings of the Workshop on Knowledge Management in Interand Intra-organizational Environments, EADS - Paris, December 5-7, 2001.

Panetto, H. (2002). UEML constructs representation. Proceedings of the workshop on Common Representation of Enterprise Models. IPK, Berlin, February, 20-22

Panetto, H., Berio, G., Benali, K., Boudjlida, N., Petit, M. (2004). A Unified Enterprise Modeling Language for enhanced interoperability of Enterprise Models. Preprints of the 11 th IFAC INCOM2004 Symposium.

Panetto, H., Berio, G., Petit, M., Knothe, T. (2003a). Enterprise integration semantics issue. Proc. International NIST/NSF Workshop on Semantics Distance, NIST, November 10-12, 2003, Gaithersburg, Maryland, USA.

Panetto, H., Bernard, A., Kosanke, K., (2003b). Product and Process models for Enterprise Integration. Proceedings of the ISPE-CE2003 conference on Concurrent Engineering: Research and Applications, Madeira, Portugal, 26-30 July, Enhanced Interoperable Systems, 747-835.

Vernadat, F.B. (1996). Enterprise Modeling and Integration: Principles and Applications, Chapman \& Hall, London.

Vernadat, F. (2002). UEML: Towards a unified enterprise modelling language, International Journal of Production Research, $\mathbf{4 0}$ (17), 4309-4321.

Vernadat, F.B. (2002). Enterprise Modeling and Integration (EMI): Current Status and Research Perspectives. Annual Reviews in Control. 26, 15-25.

Vernadat, F.B., Zeigler, B.P. (2000). B.P. New simulation requirements for modelbased Enterprise Engineering. Proc. IFAC/IEEE/INRIA Int. Conf. on Manufacturing Control and Production Logistics (MCPL'2000), Grenoble,.

Weston, R.H. (1993). Steps towards enterprise-wide integration: a definition of needs and first-generation open solutions. International Journal of Production Research, 31(9), 2235-2254.

Williams, T.J. (1994). The Purdue Enterprise Reference Architecture. Computers in Industry, 24 (2-3), 141-158.

Whitman, L. and Huff, B. (2001). On The Use Of Enterprise Models. Special Issue on: Business Process Design, Modeling, and Analysis, International Journal of Flexible Manufacturing Systems, 13(2), 195-208.

Whitman, L.E., D. Liles, B. Huff, and K. J. Rogers. (2001). A Manufacturing Reference Model for the Enterprise Engineer. Special Issue on Enterprise Engineering, The J. of Engineering Valuation And Cost Analysis, 4(1), 15-36.

Whitman, L., B. Huff, and A. Presley. (1997). Structured models and dynamic systems analysis: The integration of the IDEF0/IDEF3 modeling methods and discrete event simulation. Proc. Winter Simulation Conference, Atlanta, GA.

Yoshikawa, H. (1995). Manufacturing and the $21^{\text {st }}$ Century - Intelligent Manufacturing Systems and the renaissance of the manufacturing-industry. Technological Forecasting and social change. 49(2), 195-213. 